\title{
Infrared spectra of fayalite crystal
}

\author{
H. Suto ${ }^{1}$, C. Koike ${ }^{2}$, H. Sogawa ${ }^{2}$, A. Tsuchiyama ${ }^{3}$, H. Chihara ${ }^{3}$, and K. Mizutani ${ }^{4}$ \\ 1 Subaru Telescope, 650 North Aohoku Place, Hilo, Hawaii 96720, USA \\ 2 Kyoto Pharmaceutical University, Yamashina, Kyoto, Japan \\ 3 Osaka University, Department of Earth and Space Science, Toyonaka, Osaka, Japan \\ 4 Communications Research Laboratory, Koganei, Tokyo, Japan
}

Received 7 January 2002 / Accepted 28 March 2002

\begin{abstract}
Crystalline silicate is an important matter since the ISO discovery of crystalline silicate features in circumstellar dusty environments. Polarized infrared reflection spectra of crystal fayalite $\left(\mathrm{Fe}_{2} \mathrm{SiO}_{4}\right)$, an end member of olivine solid solution, were measured for each crystal axis in infrared region up to $100 \mu \mathrm{m}$, and the oscillator parameters of the dielectric constants for each crystal axis are derived by fitting the reflection spectra. The optical constants derived by the oscillator parameters provide a useful standard for the optical characterization of fayalite.
\end{abstract}

Key words. methods: laboratory - dust - circumstellar matter - ISM: lines and bands

\section{Introduction}

Silicate dust is the major component of interplanetary and interstellar solid particles, and the crystalline form of silicate is now believed to exist in the circumstellar regions around some of the evolved stars and pre-main sequence stars from ISO observations (Waelkens et al. 1996; Waters et al. 1996, 1998; Malfait et al. 1998; Molster et al. 1999). The ISO discovery of crystalline silicate has demanded more laboratory research of the crystalline form of cosmic dust analogue. Some of the transmission and reflection spectra of crystalline silicates have been provided from laboratory researches (Jäger et al. 1998; Koike et al. 1993, 2000a) and have given clues for the identification of the composition of crystalline cosmic dust. However the laboratory measurements have been mainly on particles, and these results depend on particle size, shape and degree of aggregation as well as are affected by the matrix material in which particle samples were embedded.

Avoiding the ambiguities inherent in particle samples, measurements of bulk crystal provide a reliable standard of optical characterization of cosmic dust analogues. We measured the reflection spectra of synthesized bulk fayalite crystal $\mathrm{Fe}_{2} \mathrm{SiO}_{4}$ for each crystal axis in infrared regions. Fayalite, the Fe rich end member of an olivine solid solution, is an important standard material to quantify the $\mathrm{Mg} / \mathrm{Fe}$ ratio from the observed olivine spectrum, as well as to study the environment of crystalline formation of cosmic dust.

Send offprint requests to: H. Suto, e-mail: suto@naoj.org
We present the derived oscillator parameters for its dielectric constant and compare the absorption spectrum of fayalite particles with the AFGL4106 ISO spectrum. The absorption spectrum of fine particles can be reproduced with the dielectric constant by assuming particle shape and size. Our parameters offer a unique set of reliable fayalite dielectric constants for the infrared region up to $100 \mu \mathrm{m}$.

\section{Reflection spectra}

Fayalite single crystal was grown by the Floating Zone method (Takei 1978). The sample dimension was $3 \mathrm{~mm} \times$ $3 \mathrm{~mm} \times 4 \mathrm{~mm}$, which was large enough to obtain the reflection spectra with good $S / N$ ratio up to $100 \mu \mathrm{m}$. After the crystal axis was determined by the X-ray diffraction method using a precession camera, the crystal was cut perpendicular to each axis, and polished for (100) (010) and (001) surfaces with diamond paste of up to $1 / 4 \mu \mathrm{m}$.

Reflections polarized parallel to the $c$ and $b$ axes were measured on the (100) surface and to the $a$ axis on the (001) surface. Infrared spectra were obtained at room temperature with a Nicolet Nexus 670 FTIR spectrometer from $6000 \mathrm{~cm}^{-1}$ to $100 \mathrm{~cm}^{-1}$ with $1 \mathrm{~cm}^{-1}$ resolution using a reflectance module of 10 degree incidence angle. A beam splitter and wire grid polarizer in $\mathrm{KBr}$ substrate were employed for the frequency range higher than $400 \mathrm{~cm}^{-1}$ and in Polyethylene substrate for the lower frequency. The absolute level of reflectance was quite stable while integrating the spectra, and the noise level of reflectivity was 
very small, i.e. equal to or less than $0.1 \%$ at wavelengths shorter than $70 \mu \mathrm{m}$. Systematic errors should be much larger, the most significant factor being the ambiguity of the reflectivity of gold mirror used for calibration of $100 \%$ reflectance. Assuming that the gold mirror absolute reflectivity is larger than $99 \%$ for the measured wavelength, our measurement assures an absolute accuracy within $1 \%$. The error on the wavelength was less than $0.01 \mu \mathrm{m}$, guaranteed by the wavelength accuracy and narrow band width of single mode HeNe laser in the Fourier spectrometer.

Reflection at wavelengths from $2.5 \mu \mathrm{m}$ to $1 \mu \mathrm{m}$ was also measured with the resolution of $0.02 \mu \mathrm{m}$ using a JASCO grating spectrometer so that we checked the spectral value for frequency at the higher end. Although the incident angle was 5 degrees for the reflectance module in the Jasco spectrometer, and different from that in the Nicolet spectrometer, the measurements by Nicolet and Jasco spectrometers were identical at the crossover wavelength around $2 \mu \mathrm{m}\left(5000 \mathrm{~cm}^{-1}\right)$.

The reflection spectra for three surfaces perpendicular to each axis are shown in Fig. 1 . The peak positions in $\mu \mathrm{m}$ and the peak reflectivities of the features are given in the first two columns of Table 1. Olivine crystal belongs to the space group $D_{2 h}^{16}$, and there are 35 IR active optical modes described from a symmetry analysis (Iishi 1978). 27 features were observed for the measured wavelength range with good $S / N$ ratio. 7,10 and 10 modes were observed for polarization parallel to the $c$ axis $\left(B_{1 \mathrm{u}}\right), b$ axis $\left(B_{2 \mathrm{u}}\right)$, and $a$ axis $\left(B_{3 \mathrm{u}}\right)$, respectively ( $B_{i \mathrm{u}}$ is the representation by factor group analysis). The remaining 8 features may be out of the wavelength range observed or too weak to be detected.

No features are observed from $1 \mu \mathrm{m}$ to $9 \mu \mathrm{m}$, where the spectra are almost flat and slightly decreasing with increasing wavelength. Several prominent and small features were observed up to $60 \mu \mathrm{m}$. Some of the features are similar to those of forsterite bulk crystal (Sogawa et al. 2002). The strongest features around $10 \mu \mathrm{m}$, which are due to the stretching modes between $\mathrm{Si}$ and $\mathrm{O}$ atoms, have the same shape and intensity as those of forsterite, but their peak positions are shifted by $0.4 \mu \mathrm{m}$ towards longer wavelengths. The strong features from $18 \mu \mathrm{m}$ to $22 \mu \mathrm{m}$, which are due to bending mode of $\mathrm{Si}$ and $\mathrm{O}$ atoms, also have a similar trend as those of forsterite, but show a longer shift of peak positions, by $2 \mu \mathrm{m}$. As for the features at longer wavelengths, which are due to translation modes, their widths are broader than those of forsterite, and no clear one-to-one correspondence between fayalite and forsterite is found any more. The uniqueness of features at longer wavelength makes them a good tool for the detection of Fe rich olivine.

Hofmeister (1997) also measured the reflectance of fayalite crystals for each polarization. Her results generally match ours, but the reflectance she found around $10 \mu \mathrm{m}$ is more than $20 \%$ smaller than ours. Also her result shows three overtone bands and one doublet, where we did not observe any clear evidences of these features.
Table 1. Peak positions and reflectivity of fayalite crystal and its oscillator parameters.

\begin{tabular}{|c|c|c|c|c|}
\hline \multicolumn{5}{|c|}{$/ / c$ axis $\left(B_{1 \mathrm{u}}\right) \epsilon_{\infty}=3.50$} \\
\hline$\lambda_{\text {peak }} \mu \mathrm{m}$ & $R_{\text {peak }}$ & $\lambda_{j} \mu \mathrm{m}$ & $\gamma_{j} \mu \mathrm{m}$ & $S_{j}$ \\
\hline 11.20 & 0.86 & 11.64 & 0.15 & 0.74 \\
\hline 20.62 & 0.79 & 21.44 & 0.44 & 0.64 \\
\hline 22.47 & 0.31 & 22.37 & 0.93 & 0.16 \\
\hline 26.49 & 0.44 & 26.94 & 1.15 & 0.37 \\
\hline 31.86 & 0.84 & 33.04 & 0.88 & 2.58 \\
\hline 40.04 & 0.39 & 39.78 & 1.26 & 0.20 \\
\hline 50.96 & 0.58 & 51.18 & 1.09 & 0.63 \\
\hline \multicolumn{5}{|c|}{$/ / b$ axis $\left(B_{2 \mathrm{u}}\right) \epsilon_{\infty}=3.35$} \\
\hline$\lambda_{\text {peak }} \mu \mathrm{m}$ & $R_{\text {peak }}$ & $\lambda_{j} \mu \mathrm{m}$ & $\gamma_{j} \mu \mathrm{m}$ & $S_{j}$ \\
\hline 10.54 & 0.55 & 10.62 & 0.06 & 0.029 \\
\hline 11.33 & 0.86 & 11.65 & 0.09 & 0.39 \\
\hline 12.09 & 0.47 & 12.10 & 0.09 & 0.12 \\
\hline 19.49 & 0.63 & 19.98 & 0.41 & 0.24 \\
\hline 21.34 & 0.46 & 21.48 & 0.66 & 0.40 \\
\hline 29.21 & 0.54 & 30.11 & 1.10 & 0.14 \\
\hline 33.89 & 0.85 & 36.76 & 1.29 & 2.25 \\
\hline 39.21 & 0.60 & 39.30 & 1.10 & 0.98 \\
\hline 55.31 & 0.63 & 55.82 & 1.31 & 0.79 \\
\hline 58.26 & 0.47 & 58.13 & 1.42 & 0.30 \\
\hline \multicolumn{5}{|c|}{$/ / a$ axis $\left(B_{3 \mathrm{u}}\right) \epsilon_{\infty}=3.55$} \\
\hline$\lambda_{\text {peak }} \mu \mathrm{m}$ & $R_{\text {peak }}$ & $\lambda_{j} \mu \mathrm{m}$ & $\gamma_{j} \mu \mathrm{m}$ & $S_{j}$ \\
\hline 10.37 & 0.84 & 10.64 & 0.12 & 0.27 \\
\hline 10.91 & 0.74 & 10.95 & 0.07 & 0.32 \\
\hline 12.06 & 0.25 & 12.05 & 0.08 & 0.03 \\
\hline 17.68 & 0.73 & 18.33 & 0.43 & 0.73 \\
\hline 21.81 & 0.12 & 21.70 & 0.50 & 0.01 \\
\hline 27.47 & 0.66 & 28.32 & 0.97 & 0.35 \\
\hline 32.51 & 0.86 & 34.30 & 1.00 & 3.10 \\
\hline 45.99 & 0.34 & 45.35 & 1.70 & 0.10 \\
\hline 55.02 & 0.49 & 55.10 & 0.90 & 0.30 \\
\hline 57.14 & 0.42 & 56.95 & 1.00 & 0.16 \\
\hline
\end{tabular}

\section{Oscillator parameters and absorption spectra}

The oscillator parameters of the dielectric constants for fayalite crystal are derived by fitting theoretical curves to the measured reflection spectra.

The dielectric constant for each axis is expressed in the oscillator model by a function of wavelength $\lambda$ as

$\epsilon(\lambda)=\epsilon_{\infty}+\sum_{j} S_{j} \lambda^{2} /\left(\lambda^{2}-\lambda_{j}^{2}-i \gamma_{j} \lambda\right)$

Here, $\epsilon_{\infty}$ is the dielectric constant at the short wavelength limit, $S_{j}, \lambda_{j}, \gamma_{j}$ are the oscillator strength, transverse optical wavelength, and dumping factor, respectively, and $i$ is the imaginary number unit. The reflectivity $R$ is calculated from $\epsilon$ as

$R=\left|\left(\sqrt{\epsilon-\sin ^{2}(\theta)}-\cos (\theta)\right) /\left(\sqrt{\epsilon-\sin ^{2}(\theta)}+\cos (\theta)\right)\right|^{2}$. 


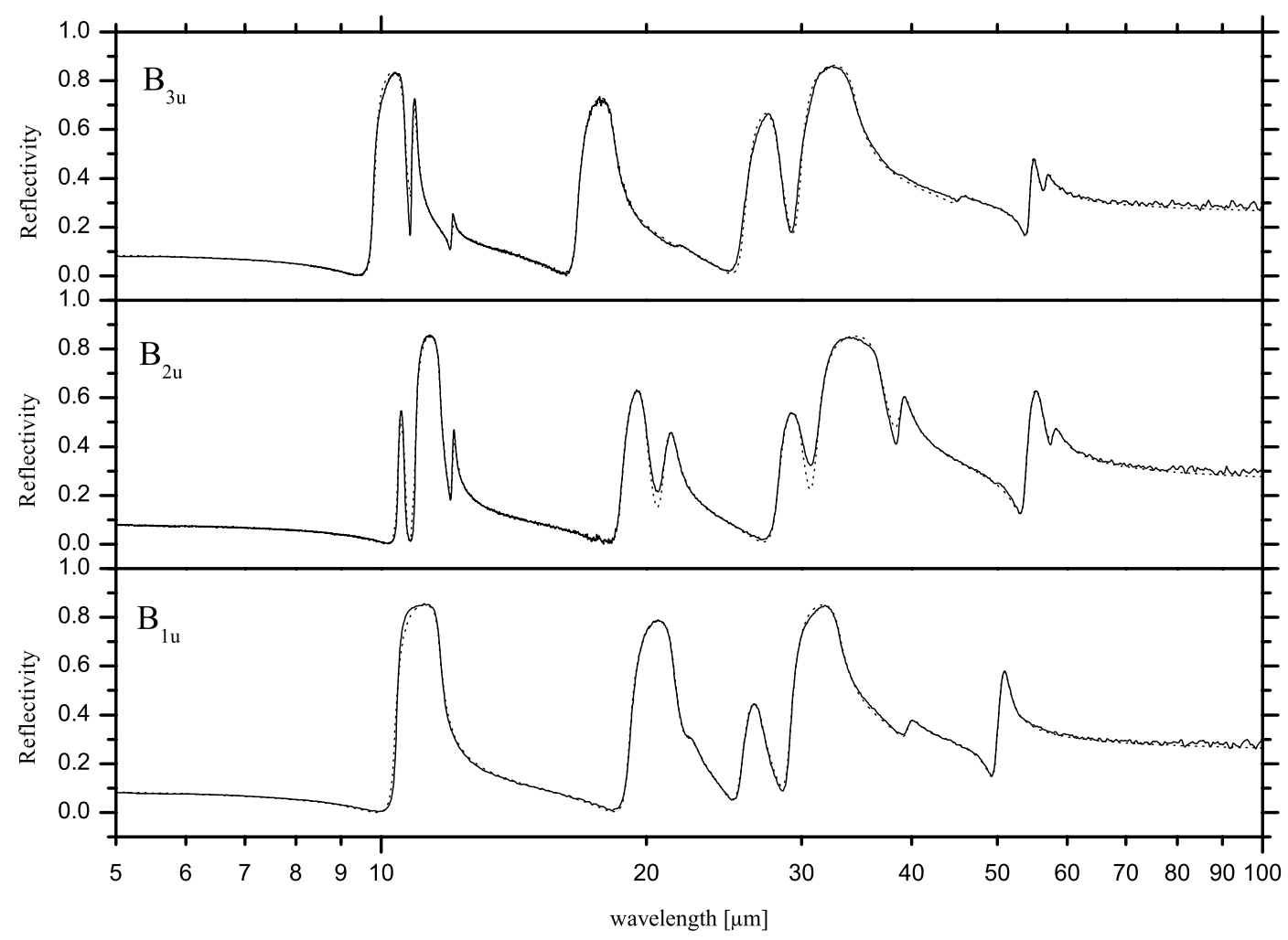

Fig. 1. Reflection spectra of fayalite for the three axies $B_{3 \mathrm{u}}$ (parallel to $a$ axis), $B_{2 \mathrm{u}}$ (parallel to $b$ axis), and $B_{1 \mathrm{u}}$ (parallel to $c$ axis). Thin lines and dotted lines are measured and calculated reflection spectra, respectively.

$\theta$ is the beam incident angle to a sample surface. In our reflectance module, the incident angle is almost normal to the surface, 10 degree.

Generally, the parameter $\lambda_{j}$ fixes the wavelength position of feature, the parameter $\epsilon_{\infty}$ determines on the intensity level of the flat portion at the shortest wavelength, the parameter $S_{j}$ is responsible for the intensity level of the features, and the parameter $\gamma_{j}$ determines the width of the features as well as their intensity. But where the features reside close to each other, the change of any parameters $\lambda_{j}, \gamma_{j}, S_{j}$ for one feature affects the shape and intensity of the others. The derived oscillator parameters are shown in Table 1. The dotted lines in Fig. 1 show the reflections calculated with parameters in Table 1 . The derived parameters reproduce the measured reflection spectra fairly well.

Assuming that the error of the measured reflection level is within $1 \%$, the ambiguity in the parameters was estimated as the differences from their original values when we resumed fitting of the parameters to the modified spectra whose level was increased by $1 \%$ with respect to the original spectra. Based on this estimation, the errors of $\epsilon_{\infty}$ and $\lambda_{j}$ are less than $1 \%$ and $0.01 \mu \mathrm{m}$, respectively. The errors of $S_{j}$ and $\gamma_{j}$ are equal to or less than three times the least digit for each values.

The mass absorption spectrum of fine fayalite particles measured by Koike (2000b) is also well reproduced with the oscillator parameters applying the CDE model as expressed in Eq. (3) below (Bohren \& Huffman 1983) where $\epsilon_{\text {med }}$ and $n_{\text {med }}$ are the dielectric and optical constants of polyethylene and $\mathrm{KBr}$ in which the particles were embedded. As shown in Fig. 2 where the thin solid line represents the absorption spectrum measured for fayalite fine particles and dotted lines the CDE model calculation (both lines are offset by 5000 for clarity), the peak positions and strengths of the fayalite absorption features are reproduced very well. This CDE model also coincides well with the transmission spectrum obtained by Jäger (1998). Based on the quite good fit to the measurements, our oscillator parameters provide reliable dielectric constants for fayalite crystal for the infrared region up to $100 \mu \mathrm{m}$, at least at room temperature.

Finally, the ISO spectrum of AFGL4106 is compared with the absorption spectra of fayalite. The absorption spectra in the Rayleigh approximation were calculated in the CDE model (Bohren \& Huffman 1983) from $\epsilon$ according to the equation,

$\kappa=6 \pi / \rho\left(n_{\text {med }} / \lambda\right) \operatorname{Imag}\left(2 \epsilon /\left(\epsilon-\epsilon_{\text {med }}\right) \log \left(\epsilon / \epsilon_{\text {med }}\right)\right)$

where $\rho$ is the density of fayalite, $4.39 \mathrm{~g} / \mathrm{cm}^{3}$, and $n_{\text {med }}$ (optical constant of medium) and $\epsilon_{\text {med }}$ (dielectric constant of medium) are set equaled to 1 (vacuum environment).

Each absorption spectrum for the three axes of fayalite crystal was calculated with Eq. (3), and averaged with equal weights to the final absorption spectrum. Figure 2 shows the absorption spectra for fayalite (solid line in the upper row) and the continuum subtracted AFGL4106 spectrum (Molster et al. 1999). The peak of AFGL4106 


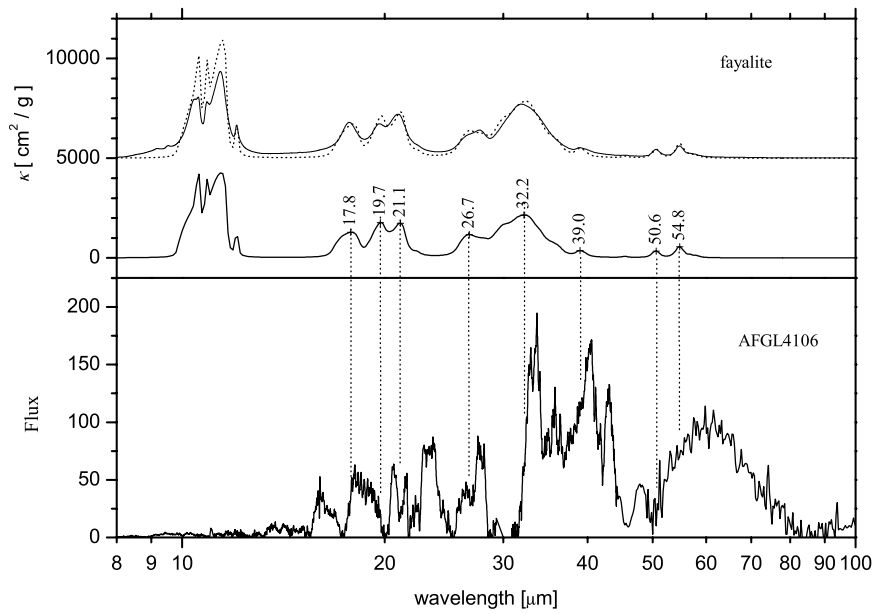

Fig. 2. Fayalite absorption spectra in the CDE model and the continuum subtracted AFGL4106 ISO spectrum (Molster et al. 1999). The solid line in the upper row is of CDE model of particles in a vacuum environment. The numbers on the solid line indicate the peak position of the features. The dotted and thin lines in the upper row are of the CDE model of particles embedded in substrate ( $\mathrm{KBr}$ and polyethylene), and of measurements of fine particles, respectively. Both lines are added offset by 5000 for clarity.

at $26.4 \mu \mathrm{m}$ may be identical to that of fayalite at $26.7 \mu \mathrm{m}$, but other features are systematically shifted from fayalite. Magnesium rich olivine and pyroxene show a better match to AFGL4106 (Jäger et al. 1998; Koike et al. 2000b), and other observational spectra (Waters et al. 1998), suggesting that the abundant form of crystalline silicate in circumstellar dusty regions is magnesium rich silicates.

As far as we know, no good match has been reported between the fayalite spectrum and ISO observational results. However, new abundant results on cosmic dust will be expected by the observations using advanced infrared facilities such as SIRTF, SOFIA, and NGST where we may discover the features of Fe rich astronomical silicates.
Our reflection data and oscillator parameters provide a useful reference for the coming research of astronomical crystal silicates.

Acknowledgements. We thank Professor. F. Takei of Osaka University for preparing fayalite crystal. We also thank Prof. L.B.F.M. Waters and Dr. F.J. Molster for providing us with ISO AFGL4106 data. We are grateful to Mr. T. Hattori for determination of crystal axis by X-ray diffraction method. We thank Prof. H. Okuda for his continuous support of our cosmic dust research.

\section{References}

Bohren, C. F., \& Huffman, D. R. 1983, in Absorption and Scattering of Light by Small Particles (Wiley-Interscience Publication), 356

Hofmeister, A. M. 1998, Phys. Chem. Minerals, 24, 535

Iishi, K. 1978, American Mineralogist, 63, 1198

Jäger, F. J., Molster, F. J., Dorschner, J., et al. 1998, A\&A, 339, 904

Koike, C., Shibai, H., \& Tsuchiyama, A. 1993, MNRAS, 264, 654

Koike, C., Tsuchiyama, A., Shibai, H., et al. 2000a, A\&A, 363, 1115

Koike, C., Chihara, H., Tsuchiyama, A., Sogawa, H., \& Suto, H. 2000b, Proc. of the 33rd ISAS Lunar and Planetary Symp., 33, 95

Malfait, K., Waelkens, C., Waters, L. B. F. M., et al. 1998, A\&A, 332, L25

Molster, F. J., Waters, L. B. F. M., Trams, N., et al. 1999, A\&A, 350, 163

Sogawa, et al. 2002, in preparation

Takei, F. 1978, J. Cryst. Growth, 43, 463

Waelkens, C., Waters, L. B. F. M., de Graauw, M. S., et al. 1996, A\&A, 315, L245

Waters, L. B. F. M., Molster, F. J., de Jong, T., et al. 1996, A\&A, 315, L361

Waters, L. B. F. M., Beintema, D. A., Zijlstra, A. A., et al. 1998, A\&A, 331, L61 